\title{
Análise dos fatores associados a quedas com fratura de fêmur em idosos: um estudo caso-controle
}

Analysis of factors associated to falls and hip fractures in elderly people: a case-control study

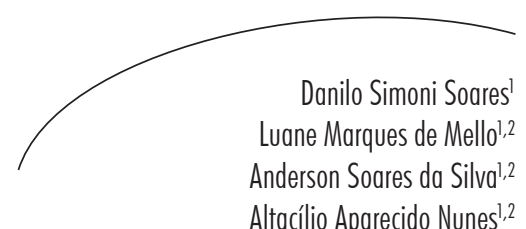

\section{Resumo}

Objetivo: Identificar os principais fatores associados a quedas e fraturas de fêmur em idosos. Métodos: Trata-se de estudo caso-controle (um grupo de casos e dois grupos controle) na proporção de 1:1:1, em que foram estudados 135 indivíduos com idade $\geq 60$ anos, pareados por sexo, no período de 2005 a 2012. Os dados foram coletados por meio de entrevistas nos domićlios dos participantes, e realizou-se levantamento de informações em prontuários. Para análise das diferenças entre proporções e médias entre os grupos, empregaram-se o teste qui-quadrado e o teste $t$ Student, respectivamente. Para o estudo da associação entre variáveis, foram realizadas análises univariadas e multivariadas empregando-se regressão logística. Como medida de efeito, foram empregados odds ratio (OR) e seu intervalo de confiança a 95\% (IC95\%). Em todas as análises, considerou-se um nível de significância de 5\%. Resultados: Após análise multivariada, os fatores de proteção contra fratura de fêmur foram: ouvir bem e possuir corrimão nas escadas de suas residências. Os fatores de risco para fratura de fêmur foram: hipertensão arterial sistêmica, sedentarismo e possuir superfície escorregadia na residência. Os fatores de proteção para queda foram: possuir corrimão nas escadas de suas residências, ser portador de osteoporose e depressão. O fator de risco de queda foi o sedentarismo. Conclusão: As fraturas de fêmur representam importante fator de morbidade em idosos. Conhecer os fatores de risco para fraturas pós-queda em idosos é essencial para o planejamento de ações individuais e coletivas voltadas à prevenção deste agravo e suas consequências. Atividades físicas, planos terapêuticos mais adequados e correção de inadequações nos domicílios desses indivíduos devem ser orientados e incentivados.
Palavras-chave: Fratura do Fêmur. Fratura do Quadril. Idosos. Estudo Caso e Controle. Brasil.

\footnotetext{
Universidade de São Paulo, Faculdade de Medicina de Ribeirão Preto, Programa de Pós-graduação em Saúde na Comunidade. Ribeirão Preto, SP, Brasil.

2 Universidade de São Paulo, Faculdade de Medicina de Ribeirão Preto, Departamento de Medicina Social. Ribeirão Preto, SP, Brasil.
} 


\section{Abstract}

Objective: To identify the main factors associated to falls and hip fractures in the elderly. Methods: This is a case-control study (a group of cases and two control groups) in a 1: 1: 1 proportion, studying 135 individuals aged $\geq 60$ years, matched by sex, from 2005 to 2012. Data were collected through interviews at the homes of participants, and information was gathered from medical records. To analyze the differences between proportions and means between groups, they employed the chi-square and Student's $t$ test, respectively. To study the association between variables, were performed univariate and multivariate analyzes using logistic regression. As effect measure, were employed odds ratio (OR) and its confidence interval at 95\% (95\%). In all analyzes, we considered a 5\% significance level. Results: After multivariate analysis, hip fracture protection factors were: listen well and have handrails on the stairs of their homes. Risk factors for hip fracture were: hypertension, sedentary lifestyle and have slippery surface in the residence. Protective factors for fall were: have handrails on the stairs of their homes, have osteoporosis and depression. Risk factor for falls was physical inactivity. Conclusion: Hip fractures are an important cause of morbidity in the elderly. Knowing the risk factors for post-fall fractures in the elderly is essential for planning individual and collective actions aimed at prevention of this disease and its consequences. Physical activities, more effective treatment plans and inadequacies correction in households of those individuals should be counseled and encouraged.
Key words: Femoral Fractures. Hip Fractures. Elderly. Case-Control Studies. Brazil.

\section{INTRODUÇÃO}

Existe grande preocupação em relação ao modo como as pessoas envelhecem atualmente. A qualidade de vida durante o envelhecimento está relacionada a perda ou diminuição da capacidade funcional do idoso, que, segundo Machado, ${ }^{1}$ é afetada diferentemente de acordo com o sexo. As mulheres idosas apresentam maior comprometimento na funcionalidade física, e os homens idosos apresentam maior comprometimento da saúde mental. ${ }^{1}$ A funcionalidade pode ser ainda mais comprometida quando o idoso sofre uma fratura de fêmur. Dados de Guimarães e colaboradores ${ }^{2}$ mostraram que $82,7 \%$ dos pacientes avaliados não necessitavam de auxílio para deambular no início do estudo. Um ano após a fratura de fêmur, esse número foi reduzido para $44,2 \%$, sendo observadas maiores dificuldades para a recuperação da marcha entre aqueles com 80 anos ou mais.
O envelhecimento pode ser influenciado por fatores biológicos, doenças e também por causas externas, entre elas a queda (Classificação Internacional de Doenças CID-10). A queda é considerada um problema de saúde pública e se dá em decorrência da perda total do equilíbrio postural, podendo estar relacionada à insuficiência súbita dos mecanismos neurais e osteoarticulares envolvidos na manutenção da postura. ${ }^{3}$ Entre as principais consequências das quedas estão as fraturas e risco de morte, o medo de cair, a restrição de atividades, o declínio na saúde e o aumento do risco de institucionalização. As quedas geram, além de prejuízo físico e psicológico, aumento dos custos com os cuidados de saúde, expressos pelo aumento da utilização de vários serviços especializados, sobretudo hospitalizações. ${ }^{4}$

Os principais fatores de risco para as quedas e fraturas são: idade, sexo, uso de drogas psicotrópicas, consumo abusivo de álcool, 
tabaco, osteoporose, menopausa precoce, sedentarismo, incapacidade física, perda do equilíbrio, perda da capacidade cognitiva e presença de comorbidades. Dentre as fraturas, as mais comuns são as de fêmur, hoje consideradas um dos maiores problemas da saúde pública, atingindo principalmente mulheres idosas. ${ }^{5}$

A osteoporose é o principal fator responsável pelo aumento na incidência de fratura de fêmur na faixa etária acima dos 60 anos. Estima-se que um terço das mulheres da raça branca com idade superior a 65 anos tenha osteoporose e $30 \%$ delas sofram ao menos uma queda por ano. ${ }^{6}$ Projeções futuras apontam que, no ano de 2050, seis milhões de pessoas sofrerão fratura de fêmur.?

Diante do exposto e da relevância do tema, o presente estudo teve como objetivo identificar os principais fatores associados a quedas e fraturas de fêmur em idosos em um município do sudeste brasileiro.

\section{METODOLOGIA}

Realizou-se estudo caso-controle (um grupo de casos e dois grupos controle) na proporção de 1:1:1, em que foram estudados indivíduos com idade $\geq 60$ anos, residentes no município de São Sebastião do Paraíso-MG, no período de 2005 a 2012. Foram considerados casos (Grupo 1), idosos que sofreram fratura de fêmur decorrente de queda, e como controles, aqueles que sofreram quedas, mas não sofreram fraturas de fêmur (Grupo 2) e aqueles que não sofreram nem queda nem fratura (Grupo 3), na proporção de 1:1:1. Optou-se pelo pareamento dos grupos quanto ao sexo devido à influência desta característica em importantes aspectos do envelhecimento. ${ }^{1}$

Considerando-se que a população de pessoas com idade igual ou superior a 60 anos em São Sebastião do Paraíso é de 13.000 indivíduos e que a prevalência de fratura de fêmur nos últimos três anos foi de 3,6/1.000 idosos, chegou-se a uma amostra mínima necessária de 33 casos e 66 controles, assumindo nível de significância de $5 \%$, estimando-se um odds ratio de 2,0 e um poder estatístico de $80 \%$. Foram excluídos do estudo os idosos que apresentaram fratura de fêmur por causas não relacionadas à queda, como acidente automobilístico.

Os dados foram coletados por meio de entrevistas estruturadas com os participantes e seus familiares, realizadas nos domicílios, e de registros em prontuários. Para a análise estatística, foram consideradas como variáveis: idade, sedentarismo (a não prática de atividade física por pelo menos 30 minutos, no mínimo três dias na semana), condição social e econômica, institucionalização, comorbidades (referidas pelos participantes do estudo e seus familiares), etnia, uso de medicamentos à época do evento, presença de osteoporose (referida pelos participantes do estudo e seus familiares), condições de habitação (presença de condições desfavoráveis no domicílio, como escadas, por exemplo), presença de cuidadores e estado mental (avaliado subjetivamente).

Para comparação de diferenças e distribuição entre proporções, foi empregado o teste quiquadrado, enquanto que para verificar diferenças entre médias foi utilizado o teste $t$ Student. Para a análise de associação entre variáveis, foram realizadas inicialmente análises univariadas e, posteriormente, análises multivariadas, empregando-se regressão logística. Como medida de efeito, foi empregado o odds ratio (OR) e seu intervalo de confiança a 95\% (IC95\%). Em todas as análises, considerou-se um nível de significância de 5\%.

O projeto de pesquisa foi aprovado pelo Comitê de Ética em Pesquisa da Faculdade de Medicina de Ribeirão Preto da Universidade de São Paulo (n ${ }^{\circ}$ 456/CEP/2011-CSE-FMRPUSP). Todos os participantes da pesquisa leram e assinaram o Termo de Consentimento Livre e Esclarecido. 


\section{RESULTADOS}

Foram avaliados 135 indivíduos, sendo 36 homens e 99 mulheres, com média geral de idade de 80,06 anos ( \pm dp 1,49), sendo 12 homens e 33 mulheres em cada um dos três grupos (casos, controle 1 e controle 2).

Os resultados do estudo mostraram que a maioria das quedas e das fraturas de fêmur ocorreu no grupo de mulheres e no domicílio (rua e quintal). Dos 135 entrevistados, apenas 20 $(15 \%)$ praticavam atividade física regularmente, e destes, apenas um $(6 \%)$ sofreu fratura de fêmur. Já entre os 115 (85\%) que não praticavam atividade física, 44 (38\%) sofreram fratura de fêmur.

As principais características socioeconômicas e epidemiológicas dos indivíduos avaliados em cada grupo são apresentadas na tabela 1.

Tabela 1. Características gerais das pessoas incluídas no estudo, considerando os três grupos (casos, controle 1 e controle 2). São Sebastião do Paraíso-MG, 2012.

\begin{tabular}{|c|c|c|c|}
\hline Variável & Casos & Controle 1 & Controle 2 \\
\hline \multicolumn{4}{|l|}{ Sexo - n (\%) } \\
\hline Masculino & $12(27)$ & $12(27)$ & $12(27)$ \\
\hline Feminino & $33(73)$ & $33(73)$ & $33(73)$ \\
\hline \multicolumn{4}{|l|}{ Idade (anos) } \\
\hline Média & 79,08 & 79,91 & 81,20 \\
\hline$\pm \mathrm{dp}$ & 7,72 & 7,45 & 7,14 \\
\hline Óbito - n (\%) & $9(20)$ & $0(0)$ & $0(0)$ \\
\hline \multicolumn{4}{|c|}{ Prática atividade física - n (\%) } \\
\hline Não & $44(98)$ & $36(80)$ & $35(78)$ \\
\hline $\operatorname{Sim}$ & $1(2)$ & $9(20)$ & $10(22)$ \\
\hline \multicolumn{4}{|l|}{ Local da queda - n (\%) } \\
\hline Rua & $8(18)$ & $16(35)$ & - \\
\hline Quintal & $16(35)$ & $12(28)$ & - \\
\hline Cozinha & $3(7)$ & $7(15)$ & - \\
\hline Quarto & $8(18)$ & $1(2)$ & - \\
\hline Banheiro & $3(7)$ & $4(9)$ & - \\
\hline Sala & $7(15)$ & $5(11)$ & - \\
\hline \multicolumn{4}{|l|}{ Causa da queda - n (\%) } \\
\hline Tontura & $11(25)$ & $4(9)$ & - \\
\hline Desequilíbrio & $14(31)$ & $8(18)$ & - \\
\hline Tropeço & $8(18)$ & $13(29)$ & - \\
\hline Animal & $1(2)$ & $1(2)$ & - \\
\hline Escada & $3(7)$ & $5(11)$ & - \\
\hline Móveis & $1(2)$ & $0(0)$ & - \\
\hline Escorregou & $7(15)$ & $13(29)$ & - \\
\hline Tapete & $0(0)$ & $1(2)$ & - \\
\hline
\end{tabular}


Foi possível observar que as principais características dos indivíduos nos três grupos são semelhantes, à exceção do sedentarismo, que só ocorreu no grupo de casos.

Com a finalidade de avaliar as possíveis associações entre as variáveis e minimizar a interferência de possíveis variáveis de confundimento, foram realizadas análises univariadas e multivariadas, respectivamente, comparando-se o grupo de casos com grupo controle 1 (tabela 2) e com o grupo controle 2 (tabela 3).

Tabela 2. Análises univariadas e multivariadas entre o grupo de casos e o grupo controle 1 (indivíduos que sofreram queda e não fraturaram o fêmur). São Sebastião do Paraíso-MG, 2012.

\begin{tabular}{lccc}
\hline \multicolumn{1}{c}{ Variável independente } & $\mathrm{n}(\%)$ & OR (IC 95\%) & $\begin{array}{c}\text { OR (IC 95\%) } \\
\text { ajustado }\end{array}$ \\
\hline Álcool (sim) & $8(8,9)$ & $1,00(0,23-4,27)$ & $1,27(0,02-8,86)$ \\
Atividade remunerada (sim) & $89(98)$ & $0,49(0,40-0,61)$ & $0,90(0,01-1,50)$ \\
Enxerga bem (sim) & $42(46,7)$ & $0,84(0,36-1,91)$ & $0,70(0,10-4,92)$ \\
Escolaridade (<8 anos de estudo) & $59(65,5)$ & $2,01(0,82-4,88)$ & $2,33(0,38-8,98)$ \\
Estado conjugal (casado) & $63(70,0)$ & $1,70(0,70-4,35)$ & $2,20(0,11-4,56)$ \\
Mora com (só) & $17(18,9)$ & $0,34(0,11-1,07)$ & $0,18(0,01-1,61)$ \\
Ouve bem (sim) & $63(70,0)$ & $0,48(0,19-1,19)$ & $0,18(0,01-0,77)$ \\
Portador de depressão (sim) & $5(5,0)$ & $1,59(0,24-9,66)$ & $1,54(0,04-6,62)$ \\
Portador de diabetes (sim) & $18(20,0)$ & $1,00(0,36-2,81)$ & $0,97(0,05-5,18)$ \\
Portador de hipertensão arterial (sim) & $57(63,3)$ & $3,66(1,47-9,13)$ & $5,20(1,15-9,40)$ \\
Portador de osteoporose (sim) & $41(45,5)$ & $0,44(0,19-1,03)$ & $0,55(0,05-6,07)$ \\
Possui animal em casa (sim) & $31(34,4)$ & $1,10(0,46-2,63)$ & $3,66(0,28-4,83)$ \\
Possui corrimão em casa (sim) & $83(92,2)$ & $0,69(0,49-1,47)$ & $0,11(0,01-0,74)$ \\
Possui degrau em casa (sim) & $22(24,4)$ & $0,79(0,30-2,06)$ & $1,64(0,19-4,10)$ \\
Possui superfície escorregadia em casa (sim) & $48(53,3)$ & $3,64(1,52-8,73)$ & $3,33(1,11-6,26)$ \\
Possui tapete em casa (sim) & $44(48,9)$ & $1,43(0,62-3,28)$ & $1,29(0,03-3,20)$ \\
Sedentarismo (sim) & $80(88,9)$ & $11,00(1,33-19,95)$ & $8,02(1,10-12,70)$ \\
Tabagismo (sim) & $16(17,8)$ & $1,83(0,26-2,71)$ & $1,98(0,18-2,38)$ \\
Tontura (sim) & $50(55,6)$ & $1,83(0,36-19,2)$ & $1,79(0,22-14,29)$ \\
Usa antidepressivos (sim) & $12(13,3)$ & $0,29(0,07-1,14)$ & $0,12(0,01-1,77)$ \\
Usa anti-hipertensivos (sim) & $59(65,5)$ & $3,83(1,50-9,75)$ & $1,82(0,01-9,17)$ \\
Usa medicamento p/ diabetes (sim) & $8(8,9)$ & $3,02(0,75-12,26)$ & $3,33(0,06-17,06)$ \\
Usa medicamentos p/ osteoporose (sim) & $6(6,7)$ & $0,48(0,08-2,74)$ & $0,12(0,01-4,42)$ \\
Usa tranquilizantes (sim) & $12(13,4)$ & $1,47(0,43-5,04)$ & $1,56(0,33-5,30)$ \\
\hline & & & \\
\hline
\end{tabular}


Como pode ser observado, a comparação do grupo de casos (quedas com fraturas) com o grupo controle 1 (quedas sem fraturas) mostrou que ter boa audição e possuir corrimão em escada no domicílio foram fatores de proteção para a não ocorrência de fratura, enquanto sedentarismo, referir ser portador de hipertensão arterial e residir em moradia com superfície escorregadia representaram fatores de risco para quedas com fraturas (tabela 2).
Ao se comparar o grupo de casos (quedas com fraturas) com o grupo controle 2 (sem quedas nem fraturas), observou-se que as variáveis associadas à proteção contra quedas foram o relato de osteoporose, bem como a presença de corrimão em escadas no domicílio. O sedentarismo e o fato de possuir tapetes no domicílio foram variáveis associadas ao risco de queda e fratura (tabela 3).

Tabela 3. Análises univariadas e multivariadas entre o grupo de casos e o grupo controle 2 (indivíduos que não sofreram queda nem fraturas). São Sebastião do Paraíso-MG, 2012.

\begin{tabular}{lccc}
\hline \multicolumn{1}{c}{ Variável independente } & $\mathrm{n}(\%)$ & $\mathrm{OR}(\mathrm{IC} 95 \%)$ & $\mathrm{OR}(\mathrm{IC}$ 95\%) ajustado \\
\hline Álcool (sim) & $6(6,7)$ & $0,48(0,08-32,74)$ & $0,24(0,01-32,53)$ \\
Atividade remunerada (sim) & $74(82,2)$ & $2,05(0,18-3,41)$ & $2,53(0,04-5,54)$ \\
Enxerga bem (sim) & $48(53,3)$ & $1,43(0,62-3,29)$ & $1,83(0,59-3,49)$ \\
Escolaridade (<8 anos de estudo) & $60(66,7)$ & $1,62(0,68-3,84)$ & $1,04(0,23-4,65)$ \\
Estado conjugal (casado) & $61(67,8)$ & $1,89(0,34-2,30)$ & $2,07(0,35-2,23)$ \\
Mora com (só) & $9(10,0)$ & $1,28(0,32-15,12)$ & $2,00(0,47-16,40)$ \\
Ouve bem (sim) & $65(72,2)$ & $1,75(0,69-4,47)$ & $2,16(0,43-5,84)$ \\
Portador de depressão (sim) & $1(1,11)$ & $0,04(0,03-0,60)$ & $0,03(0,02-0,10)$ \\
Portador de diabetes (sim) & $15(16,7)$ & $0,61(0,20-1,90)$ & $0,70(0,01-10,00)$ \\
Portador de hipertensão arterial (sim) & $53(58,9)$ & $2,31(0,98-5,47)$ & $3,03(0,13-6,52)$ \\
Portador de osteoporose (sim) & $39(43,3)$ & $0,36(0,15-0,86)$ & $0,07(0,01-0,49)$ \\
Possui animal em casa (sim) & $30(33,3)$ & $1,22(0,51-2,94)$ & $2,09(0,42-10,47)$ \\
Possui corrimão em casa (sim) & $88(97,8)$ & $0,49(0,39-0,60)$ & $0,45(0,31-0,98)$ \\
Possui degrau em casa (sim) & $17(18,9)$ & $1,55(0,53-4,52)$ & $1,22(0,23-6,51)$ \\
Possui superfície escorregadia em casa (sim) & $40(44,4)$ & $1,72(0,74-3,99)$ & $2,18(0,51-9,26)$ \\
Possui tapete em casa (sim) & $51(56,7)$ & $2,77(1,17-6,56)$ & $2,81(1,69-7,54)$ \\
Sedentarismo (sim) & $79(87,8)$ & $12,57(10,53-12,97)$ & $14,00(10,15-17,90)$ \\
Tabagismo (sim) & $9(10,0)$ & $0,25(0,05-11,29)$ & $1,07(0,09-11,92)$ \\
Tontura (sim) & $39(43,3)$ & $2,29(0,97-5,36)$ & $1,12(0,24-5,21)$ \\
Usa antidepressivos (sim) & $10(11,1)$ & $0,09(0,01-0,75)$ & $0,07(0,01-3,43)$ \\
Usa anti-hipertensivos (sim) & $55(61,1)$ & $2,35(0,99-5,62)$ & $0,69(0,01-4,51)$ \\
Usa medicamento p/ diabetes (sim) & $8(8,9)$ & $1,75(0,09-7,81)$ & $1,25(0,01-10,00)$ \\
Usa medicamentos p/ osteoporose (sim) & $12(13,3)$ & $2,22(0,62-7,98)$ & $1,77(0,21-5,20)$ \\
Usa tranquilizantes (sim) & $9(10)$ & $0,78(0,19-33,12)$ & $1,30(0,17-22,73)$ \\
\hline & & & \\
\hline & & & \\
& & &
\end{tabular}




\section{DISCUSSÃO}

O presente estudo reafirmou que as fraturas de fêmur representam importante fator de morbidade em idosos. Entre outros aspectos, constatou-se que renda, presença de corrimão nas escadas das residências e boa audição foram fatores de proteção contra a ocorrência de fratura de fêmur, enquanto que ser portador de hipertensão arterial sistêmica, fazer uso de anti-hipertensivos, ser sedentário e a presença de piso escorregadio nas residências atuaram como fatores de risco. Com relação às quedas, o sedentarismo e a presença de tapetes nas residências foram os principais fatores de risco encontrados, enquanto a presença de corrimão em escadas, ser portador de osteoporose e possuir diagnóstico (autorreferido) de depressão foram identificados como fatores de proteção.

Os idosos que declararam receber algum tipo de remuneração foram menos suscetíveis a sofrerem fratura, quando comparados com aqueles que não possuíam nenhuma fonte de renda. Este dado também foi observado por outros autores, que o atribuíram ao pequeno espaço físico e condições ambientais às quais as pessoas de menor renda estavam submetidas ${ }^{8}$ e a pior saúde óssea decorrente de condições nutricionais mais precárias. ${ }^{?}$

Os idosos sedentários também apresentaram mais chances de sofrer quedas e fraturas de fêmur, e esta maior suscetibilidade provavelmente se deve ao enfraquecimento e hipotrofia muscular decorrentes da falta de atividade física. Esse dado está de acordo com vários outros estudos da literatura, que mostraram que a prática de exercícios físicos representa um fator protetor contra quedas e fratura de fêmur, independentemente de sexo e condição socioeconômica, certamente por fortalecer e aumentar a massa muscular desses indivíduos, além de melhorar o equilíbrio corporal. ${ }^{10-14}$

As análises mostraram que a presença de superfície escorregadia aumentou as chances de queda e fratura de fêmur, representando importante fator de risco para a população estudada. Esta associação também foi observada em outros grupos. ${ }^{15-17} \mathrm{Da}$ mesma forma, possuir tapetes em suas residências esteve mais associado a quedas entre os idosos estudados. Esses dados estão de acordo com dados de outros autores e provavelmente refletem uma característica importante desses indivíduos, que tendem a passar a maior parte do tempo em suas residências e apresentar outros fatores de risco que se somam - por exemplo, a diminuição da força muscular. ${ }^{18,19}$ Por outro lado, a presença de corrimão em escadas nas residências de idosos representou um fator de proteção contra quedas, o que também foi observado em outros estudos. ${ }^{17}$

Ao analisar as condições de saúde e morbidades associadas a quedas e fraturas de fêmur, verificou-se que idosos portadores de hipertensão arterial sistêmica (HAS) e em uso de anti-hipertensivos foram mais propensos a sofrer fratura de fêmur, o que pode estar relacionado com o tipo de medicamento utilizado por esses indivíduos. ${ }^{19}$ Uma possível explicação para o aumento do risco de fratura de fêmur em portadores de HAS é o aumento na perda urinária de minerais, principalmente o cálcio. ${ }^{20}$ Solomon e colaboradores ${ }^{21}$ avaliaram o risco de fratura em relação a várias categorias de anti-hipertensivos e observaram que o uso de bloqueadores de receptor de angiotensina e os diuréticos tiazídicos estiveram associados a um menor risco, quando comparados ao uso de bloqueadores dos canais de cálcio. Os tiazídicos também estiveram associados à menor suscetibilidade a fratura de fêmur em outros estudos e uma possível explicação seria o efeito poupador de cálcio apresentado por esta família de diuréticos. ${ }^{10}$

O diagnóstico ou relato de depressão e fazer uso de medicamentos antidepressivos estiveram associados à menor propensão a quedas, diferentemente de outros estudos que relataram depressão e uso de antidepressivos como fatores de risco associados a quedas na população idosa. ${ }^{22,23}$ Kwan e outros ${ }^{22}$ acreditam que um dos motivos para isto seria o fato de as pessoas 
depressivas serem mais sedentárias, o que reduz a força muscular dos membros inferiores e favorece a diminuição do equilíbrio do corpo. Por outro lado, idosos com depressão ou em uso de medicação antidepressiva provavelmente apresentam mobilidade reduzida, expondose menos aos riscos, o que poderia explicar os achados do presente estudo. Este é um dado interessante que merece mais investigação, especialmente se for considerada a prevalência dessa doença, que frequentemente necessita de uso de medicamentos específicos para o controle adequado dos sintomas.

Ainda, os resultados das análises mostraram que idosos portadores de osteoporose sofreram menos quedas. Esta observação também está em desacordo com resultados de outros estudos, ${ }^{24-26}$ que encontraram risco maior de quedas e quedas recorrentes entre mulheres com osteoporose, o que, segundo os autores, poderia ser explicado pela presença de alterações posturais, na marcha e no equilíbrio postural apresentado por esses indivíduos.

Outra informação interessante obtida com base na análise dos resultados do estudo foi que idosos que ouviam bem tiveram chances menores de fraturar o fêmur, o que também foi observado por outros autores. Estudo realizado por Ramos e colaboradores ${ }^{15}$ avaliando idosos com história pregressa de quedas encontrou associação entre problemas auditivos e uso de aparelhos auditivos e quedas.

A grande maioria das fraturas de fêmur é ocasionada por quedas. Assim, medidas preventivas necessitam ser adotadas para que sua ocorrência seja reduzida. A prática de exercícios físicos regulares é defendida por vários autores com o objetivo de combater o sedentarismo, fortalecer e aumentar a massa muscular, além de melhorar a postura e o equilíbrio corporal desses indivíduos. A redução do uso de medicamentos psicotrópicos, tratamento adequado de depressão e hipertensão arterial, inclusive optando-se por medicamentos mais apropriados para esta população, são medidas que devem ser incentivadas. As alterações no ambiente domiciliar também devem fazer parte das orientações aos idosos, para que se evitem escorregões, tropeções e quedas. Neste sentido, eliminação de pisos escorregadios, retirada de tapetes e instalação de corrimãos nas rampas, escadas e banheiros são medidas preventivas simples e eficientes.

Ao final, por se tratar de estudo observacional do tipo caso-controle, é preciso levar em consideração a presença de limitações metodológicas, entre as quais a possibilidade de vieses (seleção, memória e informação). As informações obtidas se referiam a dados passados ou foram autorreferidas (por exemplo, informações sobre uso de medicamentos e doenças), o que certamente contou com a memória e a capacidade cognitiva do entrevistado. Muitas vezes as informações foram complementadas pelos familiares e isso também pode ter introduzido algum viés de informação.

Outra questão a ser considerada é que algumas informações foram extraídas de prontuários, o que também implica limitações quanto à qualidade desse tipo de registro. Entretanto, sabendo-se de antemão de todas essas questões, procurou-se imprimir maior rigor no momento da coleta e do tratamento estatístico dos dados. Mesmo assim, outros estudos, com metodologia menos sujeita a vieses, deverão ser conduzidos.

\section{CONCLUSÃO}

O presente estudo propiciou melhor entendimento dos fatores associados ao risco de queda e fraturas em idosos, informações fundamentais para o planejamento de ações individuais e coletivas voltadas à prevenção desse agravo e suas consequências.

Concluiu-se que a prática regular de exercícios físicos, a elaboração de planos de terapêuticos mais apropriados e incentivar a eliminação de 
inadequações nos domicílios desses indivíduos são medidas que deverão ser orientadas individualmente aos pacientes, bem como disseminadas na forma de ações direcionadas à população idosa pelos profissionais da saúde e gestores.

\section{REFERÊNCIAS}

1. Machado AM, Braga ALF, Garcia MLB, Martins LC. Avaliação da qualidade de vida em idosos pós-fratura da extremidade proximal do fêmur. Arq Bras Ciênc Saúde 2012;37(2):70-5.

2. Guimarães FAM, Lima RR, Souza ACS, Livani B, Belangero WD. Avaliação da qualidade de vida em pacientes idosos um ano após o tratamento cirúrgico de fraturas transtrocanterianas do fêmur. Rev Bras Ortop 2011;46 suppl 1:48-54.

3. Fabrício SCC, Rodrigues RAP, Junior MLC. Causas e consequências de quedas de idosos atendidos em hospital público. Rev Saúde Pública 2004; 38(1):93-9.

4. Perracini MR, Ramos LR. Fatores associados a quedas em uma coorte de idosos residentes na comunidade. Rev Saúde Pública 2002;36(6):709-16.

5. Pereira SRM, Buksman S, Perracini M, Py L, Barreto KML, Leite VMM. Quedas em Idosos. Rio de Janeiro: SBGG: 2001. Projeto Diretrizes.

6. Guarniero R, Oliveira LG. Osteoporose: atualização no diagnóstico e princípios básicos para o tratamento. Rev Bras Ortop 2004;49(9):477-85.

7. Porter RW, Miller CG, Grainger D, Palmer SB. Prediction of hip fracture in elderly women: a prospective study. BMJ 1990;301(6753):638-41.

8. Reimers A, Laflamm L. Hip fractures among the elderly: personal and contextual social factors that matter. J Trauma 2007;62(2):365-69.

9. Zingmond DS, Soohoo NF, Silverman SL. The role of socioeconomic status on hip fracture. Osteoporos Int 2006;17(10):1562-68.

10. Määttä M, Terho E, Jokinen H, Pulkkinen P, Korpelainen J, Heikkinen J, et al. Lifestyle factors and site-specific risk of hip fracture in community dwelling older women: a 13-year prospective population-based cohort study. BMC Musculoskelet Disord 2012;13:173.

11. Benetou V, Orfanos P, Benetos IS, Pala V, Evangelista A, Frasca G, et al. Anthropometry, physical activity and hip fractures in the elderly. Injury 2011;42(2):188-93.
12. Napoli N, Schwartz AV, Palermo L, Jin JJ, Wustrack $\mathrm{R}$, Cauley JA, et al. Risk factors for subtrochanteric and diaphyse al fractures: the study of osteoporotic fractures. J Clin Endocrinol Metab 2013;98(2):659-67.

13. Pinheiro M, Ciconelli RM, Martini LA, Ferraz MB. Fatores de risco para quedas recorrentes entre mulheres e homens brasileiros: o estudo brasileiro sobre osteoporose (BRAZOS). Cad Saúde Pública 2010;26(1):89-96.

14. Kannus P, Uusi-Rasi K, Palvanen M, Parkkari J. Non-pharmacological means to prevent fractures among older adults. Ann Med 2005;37(4):303-10.

15. Ramos CV, Santos SSC, Barlem ELD, Pelzer MT. Quedas em idosos de dois serviços de pronto atendimento do Rio Grande do Sul. Rev Eletrônica Enferm 2011;13(4):703-13.

16. Fhon JRS, Rosset I, Freitas CP, Silva AO, Santos JLF, Rodrigues RAP. Prevalência de quedas de idosos em situação de fragilidade. Rev Saúde Pública 2013;47(2):266-73.

17. Pinho TAM, Silva AO, Tura LFR, Moreira MASP, Gurgel SN, Smith AAF, et al. Avaliação do risco de quedas em idosos atendidos em Unidade Básica de Saúde. Rev Esc Enferm USP 2012;46(2):320-27.

18. Rosen T, Macka K, Noonan R. Slipping and tripping: fall injuries in adults associated with rugs and carpets. J Inj Violence Res 2013;5(1):61-9.

19. Butt DA, Mamdani M, Austin PC, Tu K, Gomes T, Glazier RH. The risk of hip fracture after initiating antihypertensive drugs in the elderly. Arch Intern Med 2012;172(22):1739-44.

20. Cappuccio FP, Meilahn E, Zmuda JM, Cauley JA. High blood pressure and bone-mineral loss in elderly white women: a prospective study. Study of osteoporotic fractures research group. Lancet 1999;354(9183):971-75.

21. Solomon D, Mogun H, Garneau K, Fischer MA. Risk of fractures in older adults using antihypertensive medications. J Bone Miner Res 2011;26(7):1561-67. 
22. Kwan M, Lin SI, Close JC, Lord SR. Depressive symptoms in addition to visual impairment, reduced strength and poor balance predict falls in older taiwanese people. Age Ageing 2012;41(5):606-12.

23. Eggermont LHP, Penninx BWJH, Jones RN, Leveille SG. Depressive symptoms, chronic pain, and falls in older community-dwelling adults: the mobilize Boston study. J Am Geriatr Soc 2012;60(2):230-37.

24. Silva RB, Paiva LC, Oshima MM, Morais SS, Neto AMP. Frequência de quedas e associação com parâmetros estabilométricos de equilíbrio em mulheres na pós-menopausa com e sem osteoporose. Rev Bras Ginecol Obstet 2009;31(10):496-02.

25. Glintborg B, Hesse U, Houe T, Claus MJ, Podenphant J, Zerahn B. Osteoporosis among fallers without concomitant fracture identified in an emergency department: frequencies and risk factors. Adv Orthop 2011:1-8.

26. Riera R, Trevisani VFM, Ribeiro JPN. Osteoporose: uma importância da prevenção de quedas. Rev Bras Reumatol 2003;43(6):364-68.

Recebido: 11/3/2014

Revisado: 08/1/2015

Aprovado: 24/2/2015 